\title{
La plusvalía como sistema de financiación urbana: estudio comparativo en Ciudad de México y Manizales, Colombia
}

Georgina Isunza-Vizuet. Instituto Politécnico Nacional, Ciudad de México, México. Edisson Castro. Universidad de Manizales, Manizales, Colombia. Claudia Munévar. Universidad de Manizales, Manizales, Colombia.

RESUMEN | Se presenta un análisis de los instrumentos de recuperación de la plusvalía urbana en Ciudad de México y en Manizales, Colombia. Ambos contextos, aunque heterogéneos, tienen referencias contrastables en el marco regulatorio, en los instrumentos de recaudación y en los alcances tributarios. Se parte de una discusión conceptual de la plusvalía y la revisión de experiencias internacionales en los métodos, resultados y limitaciones de los instrumentos utilizados. En seguida se desarrolla un análisis comparativo de las bases normativas e instrumentales de recuperación de la plusvalía. Finalmente, se examinan los alcances recaudatorios en las finanzas públicas locales.

PALABRAS ClAVE | ordenamiento territorial, desarrollo regional y local, mercado inmobiliario.

ABSTRACT | This article reports an analysis of surplus capture instruments in Mexico City and in Manizales, Colombia. Both contexts, although heterogeneous, have verifiable references in the regulatory framework, collection instruments and tax scopes. The paper starts by developing a conceptual discussion on surplus value, a review of international experiences concerning methods, to then expose the results and limitations of the instruments. Furthermore, we develop a comparative analysis of the normative and instrumental bases of recovery of surplus value. Lastly, we analyze the collection scopes in local public finances.

KEYWORDS | spatial planning, regional and local development, real estate market.

Recibido el 13 de septiembre de 2019, aprobado el 11 de abril de 2020.

E-mails: G. Isunza-Vizuet, gisunza@ipn.mx | E. Castro, ecastro@umanizales.edu.co|C. Munévar, cmunevar@umanizales.edu.co 


\section{Introducción}

La revisión internacional de experiencias en la captura de la plusvalía urbana por parte del Estado varía según los regímenes de propiedad, el nivel de descentralización fiscal, la autonomía territorial y las sinergias público-privadas. Existen varios instrumentos de recuperación de plusvalías cuya naturaleza se asume bien como un tributo, una regulación fiscal o una contribución especial, variantes que tienen como fundamento común la distribución equitativa de cargas y beneficios y el cumplimiento de una función social de la propiedad. Cada uno de esos instrumentos depende de la teleología normativa y la capacidad ejecutiva de los planificadores urbanos para ponerlos en práctica (Torres, 2006). En algunos de ellos se reconoce, por ejemplo, los gravámenes de arrendamientos y concesiones de largo plazo, como sucede en China, Vietnam, Hong Kong, Singapur y la India, cuyos regímenes de propiedad del suelo son públicos. Otras figuras, como las contrapartidas en especie y las cesiones de tierras, son comunes en Alemania e Israel. En otros casos se hace recuperación directa del plusvalor con cobros de valorización, pagos por recargos y reajustes, bonificaciones de densificación, cesiones por derechos de construcción, esquemas tributarios de cofinanciación e indemnizaciones por derechos de construcción o la proveeduría de bienes y servicios, como sucede en Australia y Canadá. Por último, en algunos casos las contribuciones se hacen mediante cuotas de vivienda asequible al margen de los precios del mercado, como ocurre en Inglaterra, particularmente en Londres. En otros casos, como España, existe un deber del promotor privado en cuanto a hacer cesiones de suelo en proyectos de vivienda de interés social o densificación eficiente del suelo. En este último caso, la figura de cesión de tierras aplica tanto para los promotores privados como para los públicos, especialmente en lo relativo al equipamiento de infraestructura urbana de interés común (Morell, 2014).

En México, el uso de los instrumentos señalados es aún débil, debido a problemas derivados de los arreglos políticos y los intereses de los actores que intervienen en la gestión del suelo. A tal dificultad se suma la debilidad institucional y la ineficiencia recaudatoria, características de los gobiernos municipales frente a la fuerte centralización de recursos en los gobiernos estatales y federal.

En contraste con Colombia, las disposiciones constitucionales y legales mexicanas definen un marco procedimental de captura de plusvalía a nivel nacional, que a su vez es canalizado por los entes territoriales a través de Plan de Ordenamiento Territorial (РОт), reglamentados por acuerdos o decretos. No obstante, respecto de la naturaleza conceptual y jurídica, la definición de plusvalía aún contiene imprecisiones desde la perspectiva teórica, legal e institucional.

Por otra parte, la operación de proyectos de desarrollo urbano en diferentes países está condicionada a la relación Estado-Mercado, según el nivel de autonomía, intermediación o regulación de los gobiernos locales respecto de las dinámicas urbanas; esto implica que los operadores privados determinarán, en aquellos casos donde hay menor intervención estatal, las actuaciones redistributivas para la recuperación de las plusvalías. Este es un escenario altruista. En otros casos, el Estado 
determinará estrictamente las figuras de recuperación de plusvalías y la operación de los proyectos, modalidades condicionadas al régimen de propiedad del suelo.

El objetivo de este trabajo es realizar un análisis comparativo de la normatividad que rige los instrumentos de recuperación de plusvalías en dos ciudades: la Ciudad de México (CDmx) y la ciudad de Manizales, Colombia. La primera, a pesar de la intensa actividad inmobiliaria y de la concentración de las zonas generadoras de plusvalía más dinámicas del país, carece de un marco legal-constitucional que reconozca explícitamente su captura. En contraste, en Manizales, Colombia, las disposiciones jurídicas permiten a los entes territoriales recuperar una proporción de la plusvalía, pero aún hay una limitada capacidad de gestión ejecutiva para aprovechar plenamente los recaudos de las rentas del suelo.

La postura metodológica se inscribió en el marco de la investigación comparativa, que, en el contexto de las ciencias sociales, tiene fines idiográficos o interpretativos. De acuerdo con Nohlen (2000), se trata de un procedimiento de comparación sistemática de casos de análisis, que se aplica principalmente con fines de generalización empírica y de verificación de hipótesis. Se catalogan diferentes métodos comparativos; incluso, el análisis de caso es comprendido como un método de la investigación comparativa para hacer interpretaciones valorativas sobre los hallazgos empíricos, cobrando "relevancia las propuestas que le han asignado a la comparación el estatus de método científico, específicamente en el contexto de las ciencias sociales" (Piovani \& Krawczyk, 2017, p. 824).

¿Por qué comparar la Ciudad de México con Manizales? Primero, como lo reconocen Piovani y Krawczyk (2017), los objetos de comparación requieren del trabajo colaborativo de expertos que establezcan dimensiones y propiedades que comparar. Segundo, la investigación comparativa también representa una interacción entre el argumento teórico y la evidencia empírica. En este sentido, "hay mucho que aprender de las distintas manifestaciones de mercados del suelo y de atributos de políticas del suelo en países o jurisdicciones con estructuras sociopolíticas y económicas aparentemente similares que producen distintos resultados" (Smolka \& Mullahy, 2010, p. xviii). Así, las escalas de las ciudades son contrastantes, por lo que la existencia de una disimilitud de ambientes podría ser considerada como condición necesaria para que una investigación pueda definirse como comparativa (Piovani \& Krawczyk, 2017).

La CDMx cuenta con una población cercana a 9 millones de habitantes, de acuerdo con el Instituto Nacional de Estadística y Geografía (INEgi, 2015). Pertenece a la Zona Metropolitana de la $\mathrm{CDMx},{ }^{1}$ que encabeza el sistema urbano nacional con 20,9 millones de habitantes, equivalentes al 18\% de la población nacional, y ocupa un área de $7.866 \mathrm{~km}^{2}$. Es la entidad con mayor concentración de actividad económica, que aporta el $16,5 \%$ del Producto Interno Bruto (PIB) nacional y el $23 \%$ del pib en el sector servicios, y en la cual se concentra el $40 \%$ de los corporativos, con el 57\% de empleo y el 66\% del valor agregado (INEGi, 2014; Censos Económicos 2014).

1 La Zona Metropolitana de la cDmx está integrada por las 16 alcaldías de la Ciudad de México, 59 municipios del Estado de México y uno del Estado de Hidalgo. Véase Consejo Nacional de Población (CONAPO, 2017). 
Manizales, por su parte, es una ciudad intermedia con una población de aproximadamente 400 mil habitantes, que representa cerca del $0,9 \%$ de la población colombiana, en un área de $572 \mathrm{~km}^{2}$. Se estima que el PIB del municipio es cercano a 1.375 millones de dólares anuales, y representa el 52\% del PIB departamental y el $0,76 \%$ del pib nacional. Su presupuesto es cercano a 200 millones de dólares anuales (12\% del рів municipal), de los cuales el 28,8\% corresponde a recursos de transferencias del gobierno nacional (Alcaldía de Manizales, 2017).

A pesar de su reducido tamaño frente al de la CDMx, Manizales es un buen referente para analizar la experiencia en la recuperación de las plusvalías urbanas, y ello por dos razones: primero, la dinámica de urbanización que ha experimentado en los últimos años, acompañada de un buen desempeño en la calidad de vida urbana y las condiciones de hábitat; y segundo, porque es una ciudad con capacidad institucional para generar sinergias de trabajo público-privado y ha sido pionera en el modelo de recaudos por contribuciones de valorización, que en los últimos veinte años se han traducido en obras de infraestructura urbana.

La comparación de ambas ciudades, más allá de asumir referencias de escala, busca documentar experiencias institucionales y normativas con las que se ha gestionado, en términos instrumentales y fiscales, la recuperación de plusvalías. Al mismo tiempo, existen propiedades comunes o similitudes entre ambas ciudades que dan la pauta para hablar de contextos latinoamericanos similares en cuanto a las formas de urbanización y el dinamismo inmobiliario asociados a la generación de plusvalías. Cabe señalar también que en el análisis comparativo de la cDmx y de Manizales se aplican, para ambas ciudades, algunos preceptos teórico-conceptuales sobre el potencial de valorización del suelo desde la perspectiva legal, de gestión y como fuente de financiamiento del desarrollo urbano.

En el trabajo se expone, en primer lugar, las distintas nociones del concepto de plusvalía urbana; luego se presenta la experiencia de diversas ciudades latinoamericanas en el uso de instrumentos de recuperación de plusvalías; y, finalmente, se entregan los resultados del análisis comparativo de los marcos normativos y los instrumentos para el financiamiento local vigentes en cada una de las ciudades estudiadas.

\section{Discutiendo el concepto de plusvalía urbana}

La plusvalía ha tenido tránsitos conceptuales fundamentados en las teorías económicas asociadas a la producción de bienes (Ricardo, 1958; Thorngren, 2000), donde los plusvalores no internalizados en las ganancias de los sistemas de producción configuraban pasivos económicos para una de las partes, y rentas significativas para la otra (Arnaudo, 2013).

Desde la perspectiva urbana se acunó el concepto de plusvalor para referirse al gravamen que se genera por las acciones urbanísticas del Estado (Moncayo, 2011) o por el incremento inesperado y especulativo del valor de la tierra "a través de inversiones en infraestructura o de decisiones reglamentarias sobre el uso del suelo" (Barreto, 2003, p. 11). Así, la plusvalía es el incremento en el valor del suelo derivado del proceso de urbanización. Es calificada como una externalidad positiva, en 
tanto capitaliza las rentas totales a partir de los aumentos que produce cualquier cambio en los regímenes de propiedad, uso y aprovechamiento del suelo (Alterman, 2012; Friendly, 2017).

La participación del Estado en las plusvalías es considerada una forma de internalizar las externalidades del desarrollo urbano a partir del valor capitalizable de las rentas inesperadas de la tierra con miras a proporcionar bienes públicos o destinarlas a fines sociales (Fensham \& Gleeson, 2003; McAllister et al., 2018). Así, los incrementos en el valor del suelo derivados del proceso de urbanización pasan a ser parte de la riqueza pública de una ciudad. Estos incrementos, al asociarse al Estado y su función pública, demandan una recuperación para la financiación de la gestión urbanística. Tal recuperación es entendida como el proceso por el cual el sector público obtiene la totalidad o una porción de los incrementos del valor del suelo atribuibles a los esfuerzos de la comunidad, más que a las acciones de los propietarios (Maldonado, 2007).

La participación en plusvalía es, entonces, un mecanismo que busca corregir la inequidad presente en las ciudades por causa del mercado de la tierra (Jaramillo, 2011) y la distribución de los beneficios y costos de la gestión urbana entre los sujetos partícipes en ella. Estos beneficios y costos no se limitan al sujeto activo en el marco del rol estatal o al sujeto pasivo en cuanto propietario sobre los que se generaría el cobro de los beneficios vía impuestos, sino que también involucran al sujeto colectivo que representa el interés común en materia de desarrollo urbanístico (Munévar et al., 2018). En este marco, el criterio de inequidad en la distribución configuraría un enriquecimiento ilegítimo, "que condena el incremento del patrimonio de un sujeto a expensas de otro sin que haya una causa que lo justifique" (Barreto 2003, p. 12). Esta situación genera unos beneficios exorbitantes para unos propietarios por fuera de las finalidades sociales de la inversión, cuyo fin consiste en "devolver a la comunidad el aumento en el valor del suelo productor de la inversión comunitaria” (Furtado, 2007, p. 229).

Las nociones que definen la plusvalía urbana se vinculan con la función social de la propiedad y la prevalencia del interés general sobre el particular (Munévar $\&$ Hernández, 2017), figuras que trazan un límite a las libertades del propietario y funciones externas del bien que se encuentran en el haber privado (Bonilla, 2012), fijando la teleología a la que obedece esta figura de participación. En esta línea, la recuperación de los 'incrementos inmerecidos' en el valor del suelo urbano puede hacerse indirectamente, mediante su conversión en ingresos públicos, a través de diferentes vías:

- Mediante el gravamen de impuestos sobre la propiedad en general y sobre el valor de la tierra en particular asignado a la propiedad inmobiliaria como una tributación sobre el valor de la tierra, y el incremento temporal de la tasa de impuesto a la propiedad.

- Por las contribuciones por mejoras, en México, o por valorización, en Colombia, que son instrumentos regulatorios impuestos a los propietarios del suelo que se benefician de alguna forma de la inversión pública. Dichos aportes se hacen 
en especie, o directamente mediante mejoras locales para beneficio de la comunidad.

- Mediante las exacciones, que son instrumentos regulatorios donde se obliga a los propietarios del suelo a hacer contribuciones en especie a cambio de conseguir permisos para desarrollar o construir en un determinado suelo (Smolka \& Amborski, 2003).

A pesar de que estos recursos representan formas de captura de plusvalía, las pretensiones regulatorias y conceptuales anteriormente referidas establecen características específicas que las distinguen de instrumentos directos e indirectos, o como figuras de orden tributario y de contribuciones semejantes a la valorización. Por ejemplo, las nociones que identifican la plusvalía como tributo establecen un gravamen respecto al "incremento de valor de los inmuebles que resulta de las actuaciones urbanísticas que determina la ley” (Colombia, Consejo de Estado, 2014). No obstante, existen tensiones en cuanto a equiparar estas figuras, por la destinación específica propuesta para la participación en plusvalía y por la periodicidad frente al cobro tributario. En materia de la contribución por valorización, Alfonso (2011) establece que la participación en plusvalía, "no tiene esa vinculación tan inmediata con el costo y el beneficio causado por acciones específicas”, como sí lo tiene la valorización en las acciones públicas que generan una relación "directa con su costo y con el beneficio efectivamente producido" (p. 32).

En la actualidad, los mecanismos más comunes de recuperación de plusvalía que implementan los países en el mundo varían entre derechos regulatorios y recaudos tributarios; por ejemplo, mediante proyectos de desarrollo conjunto, la venta o arrendamiento de derechos, las tasas de mejora en distritos especiales, el impuesto a la propiedad, las contribuciones por valorización zonificada, etcétera (Mathur, 2019; McAllister et al., 2018). Cualquiera que sea el instrumento, el cobro de la plusvalía tiene dos objetivos esenciales: redistribuir efectivamente el aumento del valor de los predios privados debido a inversiones públicas, y corregir los errores del mercado inmobiliario (Verduzco \& Valenzuela, 2018).

De esta manera, la participación en plusvalía "es una modalidad sui géneris de gravamen sobre la propiedad de la tierra” (Moncayo, 2011, p. 224). Esta modalidad trasciende la mirada instrumental respecto del valor objetivo del suelo al aceptarse su causación desde una perspectiva social, según la cual dicha participación es catalogada como un derecho de orden moral (Doebele, 2007), colectivo (Acosta, 2010), legítimo y público (Barco \& Smolka, 2007). En este sentido, la finalidad propuesta en la recuperación de plusvalía y participación en ella debe atender no solo a las modalidades de un gravamen económico, sino al cumplimiento de una teleología justificada desde el valor colectivo, que por medio de las acciones urbanísticas genera el plusvalor del cual se pretende hacer partícipe al interés común y que materializa los derechos a la ciudad.

Las distintas nociones de plusvalía remiten básicamente a tres perspectivas: i) la económica, relacionada con el funcionamiento del mercado del suelo y la renta urbana; ii) la jurídico-social, que asocia su recuperación con los derechos colectivos; y iii) la perspectiva que la vincula con la gestión del suelo y el ordenamiento 
territorial, para hacer posible su distribución entre los actores de la ciudad: los gobiernos locales, los desarrolladores inmobiliarios y los sujetos colectivos.

\section{Experiencias en la recuperación de la plusvalía urbana}

Las plusvalías urbanas se han utilizado en diferentes ciudades como instrumentos de planificación local en la financiación de proyectos de infraestructura como vías, redes de metro y ferrocarriles, transporte urbano, equipamientos para saneamiento básico y vivienda, dotaciones comunitarias, culturales y patrimoniales. En general, la mayoría de los países entiende la importancia de aprovechar las rentas del suelo $y$, por ende, se han esforzado por dotar a los municipios de instrumentos administrativos y legales, en la lógica de la autonomía territorial, para corregir los efectos colaterales del desarrollo urbano y atenuar las desigualdades que producen las disparidades del crecimiento en las rentas del suelo.

Los instrumentos más comunes para recuperar las plusvalías urbanas han sido las contribuciones por mejora, cuya lógica es progresiva, sobre áreas con potencial de valorización, aunque no suponen una intensión redistributiva (Brown-Luthango, 2011). Asimismo, existen otros esquemas que dependen del régimen de propiedad del suelo, de la autonomía territorial y las sinergias logradas entre el Estado y los desarrolladores urbanos. Entre dichos esquemas se reconocen los siguientes: los gravámenes por concesiones o arrendamientos de largo plazo —más comunes en países socialistas-, las cesiones de tierras o de derechos de construcción, las contrapartidas en especie y en la proveeduría de bienes y servicios, los sistemas de cofinanciamiento tripartitos, los recaudos directos del plusvalor; y en algunos casos donde el mercado de vivienda tiene amplias brechas sociales, se promueven cuotas de construcción con precios discrecionales para vivienda de interés social.

En América Latina, la recuperación de plusvalías tiene brechas importantes, debido a diversas situaciones jurídico-administrativas que intervienen en la regulación de los hechos generadores de plusvalía, en el establecimiento de los modelos de cobro y en la aplicación efectiva de los recaudos por vía ejecutiva (Contreras $\&$ Ojeda, 2019). Incluso se ha debatido la naturaleza fiscal o regulatoria que deberían tener las plusvalías, máxime cuando las disposiciones legales no otorgan suficiente poder fiscal y vinculante, o cuando los recaudos se asumen como herramientas impopulares (Quitián-Díaz \& Ruiz-Hernández, 2016). Esa fractura entre lo legislativo y lo ejecutivo ha sido el principal factor que le resta eficacia en su recuperación, porque muchas veces no basta con aplicar los instrumentos y generar los cobros, sino que se debe asumir con responsabilidad el uso de los fondos en virtud de la función social y ecológica de la propiedad (Munévar \& Hernández, 2017; Smolka \& Amborski, 2003).

Desde la perspectiva del financiamiento, se reconocen por lo menos cuatro herramientas comúnmente utilizadas en América Latina para financiar proyectos urbanos: la contribución por mejoras, las exacciones y cargos por derechos de construcción, el financiamiento por concepto de impuestos y los esquemas de reajuste de terrenos. Cada una de ellas tiene ventajas y desventajas, y su eficiencia y factibilidad 
pueden depender de múltiples variables internas y externas al proyecto mismo (Blanco et al., 2016).

En Argentina, la plusvalía se cobra por mecanismos similares a los de Colombia y Brasil, donde la compensación por mejoras y el cobro directo del plusvalor se asocia a la reclasificación de usos y los mayores aprovechamientos (Canestrato et al., 2014). Hay otros instrumentos, como las compras o expropiaciones en áreas con potencial de valorización, donde el Estado adquiere predios rurales y luego los reclasifica como terrenos urbanos con fines sociales para proyectos de vivienda de interés social, dinamizando las zonas circundantes, la base de recaudo y el número de contribuyentes (Canestrato et al., 2014; Tudino et al., 2010).

Las experiencias de recuperación de plusvalías en Argentina han sido regresivas en la distribución de fondos, ya que, como sucede en Canadá, un porcentaje muy alto de las inversiones se ha focalizado en los mismos distritos con potencial de valorización (por ejemplo, en Puerto Madero y el Parque Náutico de San Fernando en Buenos Aires), neutralizando el propósito redistributivo de la plusvalía (Friendly, 2017; Peinado et al., 2018). En otras situaciones estos esquemas no solo han sido regresivos sino también discrecionales, y han generado polémicas sobre sus alcances para combatir la segregación social y las desigualdades espaciales (Medina, 2013).

En Brasil, la recuperación de las plusvalías se rige constitucionalmente con impuestos progresivos sobre tierras baldías y mediante la regulación de los derechos de propiedad, la creación de fondos para expropiaciones y la regularización de tierras para proyectos de vivienda de interés social (Smolka, 2013). Además, se ha vinculado a los desarrolladores inmobiliarios en las contrapartidas para financiar obras de infraestructura sobre corredores periféricos y desamortizar el desarrollo hacia otros polos de urbanización. Esto ha sido una forma de combatir la endogeneidad regresiva y potenciar zonas de valorización, como ha ocurrido recientemente en Sáo Paulo, a pesar de la persistente concentración de fondos (Friendly, 2017).

La lucha contra la endogeneidad distributiva es quizá el mayor desafío mundial para administrar los recursos de la recuperación de plusvalías, porque, a pesar de los esfuerzos fiscales, hay rigideces que terminan por intensificar la segregación. Así ocurrió en Perú, donde un plan de vivienda cofinanciado por la banca privada excluyó a los sectores más populares debido a las brechas en el acceso al crédito y a la rápida valorización de la vivienda, que la hizo inasequible para dichos grupos, reconfigurando su sentido social (Calderón, 2015).

Aunque las contribuciones por mejora intensifican este fenómeno, han logrado ser la herramienta más efectiva en la recuperación de plusvalías debido al grado de aceptación social con que cuentan y al carácter funcional y vinculante que tienen sobre los espacios urbanos (Acosta, 2010). Por ejemplo, en Chile, las contribuciones por mejora han sido, a lo largo del tiempo, el instrumento más eficiente y de uso casi exclusivo en la captación de plusvalías (Cáceres \& Sabatini, 2002). También en Ecuador y Uruguay, pese al amplio marco legislativo, el instrumento que predomina es de las contribuciones por mejora. Pese a lo anterior, se estima que en varios de estos países el recaudo de las plusvalías mediante la contribución por mejora es inferior al $1 \%$ de los ingresos municipales. 
En México, la contribución por mejoras constituye el instrumento más utilizado y reconocido en la Ley de Ingresos, como parte de los ingresos municipales. Sin embargo, estos recaudos son efímeros, puesto que su cobro es temporal, al operar solo mientras se amortiza el valor de la obra pública realizada. A nivel federal se utilizan, además, las exacciones urbanas, las contrapartidas por derechos de construcción, las aportaciones privadas y otras formas de compensación o indemnización por violaciones a las normas urbanísticas (Verduzco \& Valenzuela, 2018). Por ejemplo, en el estado de México, los recaudos mitigaron los impactos de las obras viales, además de las aportaciones de mejoras por servicios ambientales y por mejora para obra pública y de beneficio social.

En Colombia, las contribuciones por mejora o valorización han sido un instrumento exitoso en varias ciudades, como Bogotá, Medellín, Manizales, Bucaramanga, Cali y Barranquilla, y ello no solo por los buenos resultados que han tenido en materia de recaudos y distribución de los ingresos, sino también por su aceptación social, factores que lo han convertido en un referente internacional sobre este modelo (Acosta, 2010). De hecho, en Bogotá los recaudos por este mecanismo representaron cerca del 0,14\% de los ingresos fiscales entre 2017 y 2018 (Gallego et al., 2015), en Cali el 0,50\%, en Manizales el 5,45\% y en Medellín el 1,34\%; incluso, en esta ciudad llegó a ser hasta el $45 \%$ de los ingresos en los años sesenta (Borrero, 2013).

Junto con lo anterior, hay otros mecanismos de carácter progresivo, como el sistema de cargas y beneficios, la delineación urbana que grava el derecho de construcción o refacción y el cobro directo de las plusvalías; este último un poco más polémico, por su ambigüedad conceptual y débil legitimidad social, pese a tener un amplio marco legal y definirse como un tributo de carácter obligatorio (Munévar \& Hernández, 2017; Munévar et al., 2018). De hecho, solo ocho ciudades capitales de Colombia regulan y aplican el derecho de participación directa en plusvalías, siendo Bogotá el más exitoso, con recaudos cercanos al $0,13 \%$ de los ingresos corrientes. El procedimiento de cobro se lleva a cabo con la expedición de las licencias de construcción, en las cuales se recupera hasta el 50\% de los aumentos en el precio del suelo, producto de los cambios normativos que hubiese establecido el gobierno local y que fueran causantes de la valorización (Vejarano, 2008).

En otras ciudades, como Cali y Pereira, si bien hay vías reglamentarias que rigen los instrumentos y los montos de participación, los recaudos son insignificantes comparados con los retornos de la dinámica inmobiliaria. Esto se debe en parte al alto índice de informalidad y a la falta de contundencia institucional para aprovechar las rentas del suelo (Garcés, 2010; Ochoa \& Rojas, 2007). En todo caso, aunque el cobro directo del plusvalor ha sido menos eficiente en Colombia que en México, las contribuciones por valorización sí han tenido buenos resultados para corregir las externalidades del desarrollo urbano, aun con los efectos adversos de la endogeneidad regresiva en la distribución de los fondos.

En este contexto, la recuperación por plusvalías en América Latina avanza a un ritmo lento en materia normativa y sobre todo en la eficiencia recaudatoria, debido al letargo ejecutivo para garantizar el cumplimiento de la ley. Y aunque existen experiencias exitosas basadas en las contribuciones por mejoras, este instrumento 
carece de una figura que procure el bienestar colectivo en materia redistributiva (Barreto, 2003). Por lo tanto, es necesario pensar la planificación urbana desde la función social del derecho a la propiedad frente el derecho a construir, de manera que las rentas del suelo contribuyan a reducir las brechas sociales que produce el crecimiento desigual al interior de las ciudades (Brown-Luthango, 2011; Morell, 2007).

\section{Comparación de instrumentos regulatorios en la recuperación de las plusvalías urbanas}

\section{Marco regulatorio nacional en México y Colombia}

México es una república representativa, democrática y federal (Constitución Política de los Estados Unidos Mexicanos, 2019, art. 40), dividida en 32 entidades federativas (art. 43). En la CDMx (hasta enero de 2016 Distrito Federal) residen los Poderes Federales (art. 44). La Constitución Política de los Estados Unidos Mexicanos (CPEUM) tiene la máxima jerarquía en el marco jurídico mexicano.

Respecto de la gestión y derechos de propiedad del suelo, la CPEum estipula que la nación es propietaria original de las tierras y aguas; que tiene el derecho de imponer a la propiedad privada las modalidades que dicte el interés público; de regular en beneficio social el aprovechamiento de los elementos naturales susceptibles de apropiación para una distribución equitativa de la riqueza pública; cuidar de su conservación; lograr el desarrollo equilibrado del país y el mejoramiento de las condiciones de vida de la población rural y urbana. También puede limitar la propiedad privada para reservarse el dominio de ciertos recursos naturales, o extinguirla por causas de utilidad pública mediante la expropiación. Esto es para los usos, reservas y destinos específicos del suelo y para efectos de planeación (art. 27).

La CPEUM también faculta al gobierno a emplear instrumentos de planeación urbana derivados del Plan Nacional de Desarrollo (art. 26), establece los principios en materia fiscal (art. 31), señala las competencias en desarrollo urbano de los municipios (art. 115) y de la CDMX (art. 122). No contiene ninguna disposición explícita referida a la recuperación de plusvalía urbana; sin embargo el artículo 27 proporciona el fundamento para instituir en México la diferencia entre la propiedad del suelo y el derecho a edificar, que deriva de una potestad pública de regular el desarrollo urbano (Azuela, 2016).

Respecto de los gobiernos locales, las facultades de los municipios más importantes son formular, aprobar y administrar la zonificación y planes de desarrollo urbano municipal; participar en la creación y administración de sus reservas territoriales; participar en la formulación de planes de desarrollo regional; autorizar, controlar y vigilar la utilización del suelo, en el ámbito de su competencia, en sus jurisdicciones territoriales; intervenir en la regularización de la tenencia de la tierra urbana; otorgar licencias y permisos para construcciones; participar en la creación y administración de zonas de reservas ecológicas y en la elaboración y aplicación de programas de ordenamiento en esta materia; intervenir en la formulación y aplicación de programas de transporte público de pasajeros cuando aquellos afecten su ámbito territorial; celebrar convenios para la administración y custodia de las zonas 
federales (art. 115, fracción v). Tampoco en este nivel existen medidas explícitas para la recuperación de plusvalía urbana.

La CPEUM reconoce dos figuras tributarias tradicionales, el impuesto predial y el de adquisición de inmuebles, además de los derechos por los servicios provenientes de división e integración de la propiedad inmueble y, en algunos casos, impuestos o contribuciones especiales por mejoras o incremento de valor derivadas de las acciones, obras y servicios públicos. Por su parte, el Código Fiscal de la Federación (2019, art. 2) estipula que las contribuciones de mejoras las pagan quienes se benefician directamente de las obras públicas.

Por su parte, en Colombia, como república unitaria, la Constitución Política de 1991 estableció la plusvalía como un mecanismo para que la sociedad, en representación del Estado, participe del plusvalor que generan las diferentes acciones urbanísticas de las entidades públicas (art. 82) y así, en correspondencia con los principios señalados en la Ley de Desarrollo Territorial (LDT), 388 de 1997, garantizar la distribución equitativa de las cargas y beneficios en el marco de la función social de la propiedad y la prevalencia del interés general (capítulo IX, arts. 73 al 90). Esta ley delimitó los factores concernientes al cobro y la implementación de la plusvalía, estableciendo las destinaciones del recaudo y la competencia de los Concejos Municipales para su regulación por medio de acuerdos municipales.

Respecto de los hechos generadores de plusvalía, la LDT establece que son decisiones administrativas asociadas a acciones urbanísticas para un "uso más rentable, o bien incrementar el aprovechamiento del suelo permitiendo una mayor área edificada" (art. 74). La ley delimita, en este mismo artículo, tres hechos generadores de plusvalía: la incorporación de suelo de expansión urbana, la modificación de uso del suelo y la autorización de mejor aprovechamiento por índice habitacional o de construcción (arts. 75, 76 y 77). Así mismo, respecto al monto de participación, el artículo 79 establece que este podrá variar del 30\% al 50\%, según lo determinen los Concejos Municipales o por iniciativa del alcalde.

Atendiendo a las características propias de la participación de plusvalía, y según los hechos generadores mencionados, la Ley 388 estableció siete destinaciones específicas orientadas al interés común, relacionadas principalmente al mejoramiento de infraestructura vial; a equipamientos urbanos, de transporte y servicios públicos, así como a la ejecución de proyectos para la recreación, tales como parques o zonas verdes; y a compra de predios para desarrollar planes de viviendas de interés social, entre otros.

En ejercicio de la potestad del Gobierno Nacional, se reglamentó el Decreto 1.077 de 2015, el cual da una base jurídica para que los Concejos Municipales expidan el acuerdo que regula los aspectos procedimentales del recaudo de la participación en plusvalía. Basados en las facultades dadas por la LDT, cada municipio debe realizar un рот en el cual, además de diferentes aspectos de urbanización y organización del territorio, se debe contemplar los mecanismos de financiación; entre ellos, la participación en plusvalía. En este se debe establecer el monto de participación, los hechos generadores, momentos de exigibilidad y las zonas objeto de plusvalía.

Sin embargo, en Colombia la eficacia de esta regulación no se cumple, toda vez que, de las treinta y dos ciudades capitales, al año 2020, veinticuatro han regulado 
la figura, pero solo nueve aplican esa norma. En otras palabras, a pesar de contar con un marco regulatorio amplio, únicamente el $66 \%$ de las ciudades regula la participación en la plusvalía, pero de ellas, solo el $25 \%$ aplica dicha regulación. Así, el escenario de ineficacia normativa es representado por el $75 \%$ de las ciudades capitales. En ellas, pese a contar con una legislación nacional y, a la vez, una normatividad local, la participación en plusvalía carece de eficacia respecto a su cumplimiento.

\section{Instrumentos de la regulación local de la plusvalía urbana:}

\section{Ciudad de México y ciudad de Manizales}

En septiembre de 2017 entró en vigor la Constitución Política de la Ciudad de México (СРCDMx), que establece nuevas facultades a la Ciudad y a las alcaldías, donde se reconoce que el Gobierno de la Ciudad será el responsable de administrar y gestionar el suelo para garantizar la distribución equitativa de las cargas y los beneficios del desarrollo urbano.

El artículo 16 de la CРCDMx, establece la obligación "de los propietarios de desarrollos inmobiliarios de pagar una compensación monetaria para mitigar el impacto urbano y ambiental", con el fin de contribuir al desarrollo y equipamiento urbano. El artículo 15 estableció "la fórmula y criterios para la aplicación de dichos ingresos en las zonas de influencia o de afectación, en condiciones de equidad, transparencia y rendición de cuentas", correspondiendo a los programas y planes parciales contemplar los cambios de los usos del suelo.

La CDMX es la entidad más regulada con instrumentos para la gestión del suelo, al tener el mercado inmobiliario más dinámico del país. Existen veintinueve Normas Generales de Ordenación (NGO), que regulan la intensidad, ocupación y formas de aprovechamiento del suelo y el espacio urbano, así como las características de las edificaciones, la transferencia de potencialidades de desarrollo urbano y el impulso de la vivienda de interés social y popular. Sin embargo, en los hechos existe gran fragmentación entre lo dispuesto por los programas delegacionales o parciales y las NGO. En especial, los polígonos de actuación, instrumento que posibilita la relotificación y relocalización de usos de suelo y destinos en uno o más predios, se han convertido en un poderoso recurso de los desarrolladores inmobiliarios para obtener altas plusvalías e incumplir las normas. Una auditoría realizada en mayo de 2019 por la Secretaría de Desarrollo Urbano y Vivienda (SEDuvi) señala que al menos 48 de 174 dictámenes autorizados durante los dos últimos años presentan irregularidades, como asignación de un mayor potencial de construcción, determinación de límites de zonificación que originan una mayor área habitacional a favor del particular o a favor del proyecto, autorizaciones de mayores niveles de construcción, ampliación de la superficie de desplante y relocalización de usos del suelo entre las alcaldías.

Las obras de mitigación urbana a las que se comprometen los desarrolladores inmobiliarios son difíciles de evaluar, porque generalmente utilizan la figura del fideicomiso privado como forma de financiamiento, información no disponible por el principio de protección de datos bancarios.

En general, las zonas con mayor plusvalía urbana de la ciudad han generado procesos de gentrificación evidentes, con casos emblemáticos como el corredor Granada-Polanco, el parque La Mexicana en Santa Fe, ambos basados en un Sistema 
de Actuación por Cooperación y en donde se registran los precios más elevados del país, que fluctúan entre 200 mil y 325 mil dólares por metro cuadrado.

Cabe aclarar que las nuevas urbanizaciones en CDMX son limitadas, por varias razones: a pesar de que el 99\% de su población es urbana, el 59\% de su superficie, compuesto por cuatro alcaldías al sur de la ciudad, está catalogado como suelo de conservación. El resto de la superficie está urbanizado, con altos niveles de densidad. Las zonas rurales localizadas en las alcaldías del sur de la ciudad ejercen una gestión comunitaria de sus recursos. En este marco, el capital inmobiliario se dirige a la periferia metropolitana, principalmente a zonas rurales del estado de México que disponen de suelo urbanizable a bajo costo, propiedad de productores rurales arruinados. El mecanismo de obtención de suelo público destinado a equipamiento urbano fluctúa entre el $15 \%$ y $30 \%$ de la superficie urbanizable, dependiendo de la normatividad local. Fuera de esto, no existe en México una distribución entre el sector público y privado por recuperación de plusvalía, a diferencia del caso colombiano.

Por otro lado, la ciudad de Manizales, desde el año 2005 y conforme al artículo 82 de la Constitución Política y la Ley 388 de 1997, ha pretendido la regulación de la plusvalía directa por medio de los рот y de acuerdos municipales (por ejemplo, Acuerdo 074 de 2013 y 663 de 2007), pero está pendiente la aprobación de dichas reglamentaciones por parte del Concejo Municipal. Solo hasta el año 2017, el рот (Acuerdo 0958 de 2017) contempló su regulación. A la fecha, el рот de la ciudad de Manizales tiene establecido un porcentaje del 50\%, fijó las zonas de expansión, determinó los hechos generadores de plusvalía, estableció las respectivas destinaciones en correspondencia con la ley nacional y otorgó un plazo a la alcaldía para su reglamentación y aplicación. No obstante, las disposiciones del рот no han sido reglamentadas y la ciudad de Manizales hace parte del $75 \%$ de las ciudades de Colombia que presenta una ineficacia normativa en el marco de aplicación para el cobro directo de la plusvalía.

No obstante lo anterior, y pese a no contar con el marco de aplicación específico establecido en la Ley 388 de 1997, Manizales constituye un modelo exitoso de recuperación de plusvalías indirectas en Colombia, ejercicio que cumple a través de elementos de compensaciones asociadas a instrumentos de gestión y financiación, como el cobro por valorización, entre otros (Acosta, 2010).

\section{Financiamiento de desarrollo urbano}

En México la única fuente de financiamiento urbano que aparece registrada en las finanzas públicas municipales es la contribución de mejoras; sin embargo, representa menos del $1 \%$ de los ingresos municipales. Una diferencia importante con Colombia radica en que las fuentes de financiamiento local están constituidas por recursos federales (aportaciones y participaciones de distintos fondos del Ramo 23 y del Ramo 33). ${ }^{2}$

2 El Ramo 23, o Provisiones salariales y económicas, es un instrumento de política presupuestaria para atender las obligaciones del Gobierno Federal, cuyas asignaciones no corresponden al gasto directo de las Secretarías o sus entidades [https://ramo23.com/]. El Ramo 33, o Aportaciones Federales para Entidades Federativas y Municipios, es el mecanismo presupuestario diseñado para transferir a los estados y municipios recursos que les permitan fortalecer su capacidad de respuesta y atender demandas de gobierno en los rubros de educación, salud e infraestructura básica [https://www.ramo33.com.mx/]. 
Un somero análisis de la participación de la contribución de mejoras en México muestra el poco peso que tiene sobre los ingresos municipales, ya que en 2018 fue de solo 0,11\% (Inegi-Finanzas Públicas Estatales y Municipales, 2019). En contraste, las participaciones federales representan el 33,3\% y las aportaciones federales, el 43\%; sumando ambos conceptos, representan el $76 \%$, lo que revela la alta dependencia financiera de los municipios.

Por su parte, la Ley de Ingresos de CDMx 2019 establece que la política fiscal se enfocará al fortalecimiento de las fuentes de ingresos de la Hacienda Pública Local, con un enfoque inclusivo que garantice la continuidad de beneficios fiscales para los grupos más vulnerables y el mantenimiento de su posición como la entidad con mayor autonomía financiera del país, por su capacidad para generar ingresos propios. Ello le permitirá contar con los recursos necesarios para continuar y ampliar sus programas sociales, la prestación de servicios públicos y el mejoramiento de la infraestructura de la Ciudad, entre otros.

En CDMx, el ingreso por contribución de mejoras solo se registra desde hace algunos ańos y es poco significativo. En el periodo 2000-2018 se registra información para los años 2000, con el $0,002 \% ; 2005$, con el 0,002\%; 2012, con el $0,56 \%$; y 2013, con $0,43 \%$. En contraste, las participaciones federales representan alrededor del $40 \%$; las sigue el cobro de impuestos, cuyo aporte fluctúa entre $20 \%$ y $24 \%$, y los derechos, que aportan entre $12 \%$ y $16 \%$ del ingreso total.

En Colombia, con referencia a la captura de plusvalías, hay un rezago en el acato de los mecanismos que define la ley, especialmente en el cobro directo del plusvalor. La contribución por valorización ha sido más eficiente y en algunas ciudades se opera parcialmente por medio de instituciones descentralizadas promotoras del desarrollo urbano. Así ocurre en Bogotá, donde se hace a través del Instituto de Desarrollo Urbano (IDU); en Medellín, por medio del Fondo de Valorización del Municipio de Medellín (fonvalmed); y en Manizales, mediante el Instituto de Valorización de Manizales (Invama). En promedio, entre 2017 y 2018, en ocho ciudades donde se regula la plusvalía directa o por cobros de valorización, los índices de recaudo fueron del 1,23\% del total de ingresos; y en el cobro directo de las plusvalías, del 0,06\%.

Manizales ha sido líder en la recuperación de plusvalías por contribuciones a la valorización, las cuales han servido para potenciar inversiones en infraestructura de transporte, parques, senderos peatonales y obras de estabilización geotécnica. Los recaudos por contribuciones a la valorización han sumado, entre 2017 y 2018, cerca del $5,45 \%$ de los ingresos totales, mientras que en los cobros directos de las plusvalías no se ha causado ningún recurso, porque a pesar de que se regula, aún no se aplica como lo define la Ley 388 de 1997. En Colombia, adicionalmente, se grava el derecho a construir, que comprende la delineación urbana que aplica para la expedición de licencias de construcción, adecuación, modificación, cerramiento, reforzamiento, restauración o demolición de un inmueble. Este rubro suma cerca del $0,45 \%$ de las rentas municipales en las principales ciudades de Colombia, y en Manizales equivale al $0,57 \%$.

En general, entre 2017 y 2018, las principales rentas del suelo y el derecho de propiedad en Colombia sumaron cerca del $15 \%$ de los ingresos totales municipales, 
donde el predial y las contribuciones por valorización tuvieron la mayor importancia. No obstante, los recaudos tácitos de las plusvalías tienen una participación marginal inferior al 1\%, como también ocurre en México. Esto supone una debilidad en la obtención de rentas recurrentes, las cuales han mostrado gran potencial para subsidiar el desarrollo urbano en otras ciudades del mundo; por ejemplo, en Hong Kong son del 27\%, en Australia entre el 6\% y el 7\%, en Singapur de 2\%, en Nueva Delhi entre el 1,5\% y el 2\% y en São Paulo más del 1\% (Fensham \& Gleeson, 2003; Friendly, 2017; Hui et al., 2004; Mathur, 2019).

A pesar del rezago aludido en el caso colombiano y la debilidad normativa en México, es claro que la participación en plusvalía constituye un poderoso instrumento de financiamiento del desarrollo urbano. En este sentido, existen avances regulatorios respecto de las disposiciones legales que, si bien sientan las bases para recuperar los incrementos en el valor del suelo, pierden su efectividad en un proceso de gestión fragmentado y en las irregularidades en el cumplimiento de la norma.

\section{Conclusiones}

El análisis comparativo expuesto permite extraer importantes conclusiones. En primer lugar, en México existe un débil marco regulatorio urbano, especialmente en los gobiernos locales, para administrar el derecho a la edificabilidad. A ello se suma la complejidad adquirida en los procesos de gestión del suelo, derivada del uso de normas de ordenamiento territorial, reglamentos y áreas de actuación que ofrecen una variedad de recursos legales. Estos recursos, sin embargo, si bien dotan de instrumentos para capturar una parte del incremento en el valor del suelo, se caracterizan por un alto grado de discrecionalidad y escasa rendición de cuentas, que constituyen obstáculos para conocer el monto y destino de la plusvalía recuperada. El único instrumento reconocido legalmente es la contribución de mejoras (equivalente a la contribución por valorización en Colombia), pero es una fuente de ingresos municipales ínfima y efímera.

En cambio, Manizales se rige por un sistema centralizado y unitario, lo que permite un mandato constitucional obligatorio para los municipios. Estos, no obstante, a pesar de contar con un amplio marco jurídico, carecen de fuerza procedimental para lograr verdaderas figuras de apalancamiento del desarrollo con los fondos que resultan del dinamismo inmobiliario.

Un segundo aspecto necesario de considerar es que, aunque en México existe un sistema de gobierno federalizado, la asignación de recursos para el financiamiento local se sigue concentrando en participaciones y aportaciones federales; es decir, el ejercicio del presupuesto está muy centralizado. En Manizales, pese a la regulación nacional, en materia presupuestal corresponde a los entes territoriales la administración y ejecución de los fondos; pese a ello, los recursos por captura de plusvalía se reflejan por vía de la aplicación de instrumentos indirectos, rezagando el alcance de la regulación especial que consagra el municipio a través de su рот.

En tercer lugar, en México prevalece una fragmentación de instrumentos de planeación y ordenamiento urbano, agravada por las escasas capacidades técnicas del personal involucrado en la gestión urbana, la ineficiencia recaudatoria y la alta 
dependencia financiera de los municipios respecto de los recursos federales. Una situación similar se da en la ciudad de Manizales, donde, a pesar de las normas generales y locales, las dificultades técnicas no previstas normativamente evidencian las debilidades instrumentales manifiestas en la recuperación de plusvalías.

En síntesis, las pretensiones regulatorias de la plusvalía como fuente de financiamiento de la gestión urbana siguen congregando los esfuerzos legislativos y políticos de ciudades latinoamericanas que evidencian, por una parte, un acelerado crecimiento urbano, pero que, por otra parte, carecen de sinergias público-privadas para su participación. Esto se debe a una fragmentación entre las competencias legislativas por nivel de gobierno (nacional, subnacional y local) y entre agencias que intervienen en los procesos de gestión del suelo para ejecutar los proyectos y hacer efectivo el recaudo y la redistribución. Tal arraigada situación pone en tela de juicio la función social de la propiedad para combatir las injusticias espaciales y las brechas de crecimiento, y garantizar los derechos a la ciudad.

No obstante sus limitaciones, el contexto histórico de la captura de plusvalía en ambas ciudades, ante el escenario descrito, refleja experiencias que potencian este instrumento en elementos asociados: la dinámica de crecimiento urbano en las ciudades latinoamericanas y las capturas indirectas de plusvalía como formas de ejecuciones presupuestales inmediatas, entre otros aspectos que legitiman la participación en la plusvalía como un instrumento eficaz para reducir la inequidad en la distribución del suelo y para la financiación del desarrollo urbano.

\section{Referencias bibliográficas}

Acosta, P. (2010). Instrumentos de financiación del desarrollo urbano en Colombia: la contribución por valorización y la participación en plusvalías. Lecciones y reflexiones. Desafios, 22(1), 15-53. https://revistas.urosario.edu.co/index.php/desafios/article/ view/1289

Alcaldía de Manizales. (2017). Presupuesto general de rentas y gastos para la vigencia fiscal de 2018, Decreto 0991 de diciembre de 2017. http://www.manizales.gov.co/ RecursosAlcaldia/201801051601538370.pdf

Alfonso, O. (2011). La cuestión de las plusvalías urbanas; viejas discusiones, nuevas perspectivas. En S. Jaramillo, V. Moncayo \& O. Moncayo (Eds.), Plusvalías Urbanas. Fundamentos económicos y jurídicos (pp. 27-58). Universidad Externado de Colombia, Bogotá.

Alterman, R. (2012). Land use regulations and property values: The 'windfalls capture' idea revisited. En N. Brooks, K. Donaghy \& G.-J. Knaap (Eds.), The Oxford Handbook of Urban Economics and Planning (pp. 755-786). Oxford University Press. http://dx.doi. org/10.1093/oxfordhb/9780195380620.013.0034

Arnaudo, F. (2013). Teoría de la Plusvalía en Marx. Revista Cultura Económica, 86, 42-49. https://repositorio.uca.edu.ar/handle/123456789/2111

Azuela, A. (2016). Para una ciudad incluyente y sustentable, otro régimen de propiedad. Revista Direito \& Práxis, 7(14), 588-608. https://www.e-publicacoes.uerj.br/index.php/ revistaceaju/article/view/22949 
Barco, C. \& Smolka, M. (2007). Desafíos para implementar la participación de plusvalías en Colombia. En M. Smolka \& L. Mullahy (Eds.), Perspectivas urbanas. Temas criticos en politicas de suelo en América Latina (pp. 222-228). Lincoln Institute of Land Policy.

Barreto, N. (2003). La Plusvalía como contribución especial con fines urbanísticos. Derecho Fiscal, 1(1), 9-35. https://revistas.uexternado.edu.co/index.php/fiscal/article/view/2666

Blanco, A., Fretes, V. \& Muñoz, A. (2016). Expandiendo el uso de la valorización del suelo. La captura de plusvalías en América Latina y el Caribe. División de Gestión Fiscal y Municipal, Gerencia de Instituciones para el Desarrollo, Banco Interamericano de Desarrollo.

Bonilla, D. (2012). El liberalismo y la propiedad en Colombia. En M. Rengifo \& J. F. Pinilla (Eds.), La ciudad y el derecho. Una introducción al derecho urbano contemporáneo (pp. 153-192). Universidad de los Andes y Universidad Temis, Colombia.

Borrero, O. (2013). Contribución de valorización o mejoras en Colombia. Análisis de la experiencia colombiana. Documento de Trabajo del Lincoln Institute of Land Policy. https://www. lincolninst.edu/sites/default/files/pubfiles/borrero-wp14ob1sp-full_0.pdf

Brown-Luthango, M. (2011). Capturing land value increment to finance infrastructure investment-possibilities for South Africa. Urban Forum, 22, 37-52. https://link. springer.com/article/10.1007/s12132-010-9108-3

Cáceres, G. \& Sabatini, F. (2002). Recuperación de plusvalías: reflexiones sobre su posible aplicación en las Ciudades chilenas. Urbano, 5(6), 56-61. http://revistas.ubiobio.cl/ index.php/RU/article/view/602

Calderón, J. (2015). Programas de vivienda social nueva y mercados de suelo urbano en el Perú. EURE, 41(122), 27-47. http://dx.doi.org/10.4067/S0250-71612015000100002

Canestrato, M., Guardia, C. \& Layus, E. (2014). Discusiones en torno a la recuperación de plusvalías urbanas: análisis de instrumentos en el Municipio de General Pueyrredón. PAMPA, 10, 131-158. https://doi.org/10.14409/pampa.v1i10.4534

Código Fiscal de la Federación. (2019). http://www.diputados.gob.mx/LeyesBiblio/pdf/8_ 160519.pdf

Colombia, Consejo de Estado. (2016). Sentencia 2012-00458 de 03 de agosto de 2016. Hechos generadores de la contribución de participación en la plusvalía... Magistrado ponente, Martha Teresa Briceño de Valencia. http://legal.legis.com.co/document/Index?obra=j urcol\&document=jurcol_c40bf748ab6f4a4bacf4cd31224875c9

Consejo Nacional de Población (CONAPo). (2018). Delimitación de las zonas metropolitanas de México 2015. https://www.gob.mx/conapo/documentos/delimitacion-de-las-zonasmetropolitanas-de-mexico-2015.html

Constitución Política de la Ciudad de México. (2017). http://www.infodf.org.mx/documentos pdf/constitucion_cdmx/Constitucion_\%20Politica_CDMX.pdf

Constitución Política de los Estados Unidos Mexicanos. (2019). http://www.diputados.gob. mx/LeyesBiblio/pdf/1_060619.pdf

Contreras, M. \& Ojeda, J. (2019). Recuperación de plusvalías: herramienta impuestaria para Gobiernos Autónomos. El caso ecuatoriano. Bitácora Urbano Territorial, 29(1), 101108. https://revistas.unal.edu.co/index.php/bitacora/article/view/64548

Doebele, W. (2007). La recuperación de las plusvalías socialmente creadas en Colombia. En M. Smolka \& L. Mullahy (Eds.), Perspectivas urbanas. Temas criticos en politicas de suelo en América Latina (pp. 205-210). Lincoln Institute of Land Policy. 
Fensham, P. \& Gleeson, B. (2003). Capturing value for urban management: A new agenda for betterment. Urban Policy and Research, 21(1), 93-112. https://www.tandfonline.com/ doi/abs/10.1080/0811114032000062164

Friendly, A. (2017). Land value capture and social benefits: Toronto and São Paulo compared, IMFG. Papers on Municipal Finance and Governance, 33, 1-50. https://munkschool. utoronto.ca/imfg/uploads/431/imfgpaper_no33_land_value_capture_abigail_ friendly_july_12_2017.pdf

Furtado, F. (2007). Reformulación de las políticas de recuperación de plusvalías en América Latina. En M. Smolka \& L. Mullahy (Eds.), Perspectivas urbanas. Temas críticos en politicas de suelo en América Latina (pp. 229-235). Lincoln Institute of Land Policy.

Gallego, J. M., Gutiérrez, L. H., Ramírez, M. \& Sepúlveda, C. E. (2015). Subsidios y contribuciones. Balance financiero de los hogares bogotanos, Alcaldía de Bogotá. Alcaldía Mayor de Bogotá, Secretaría Distrital de Planeación.

Garcés, A. (2010). La informalidad urbana y los instrumentos de gestión del suelo. Caso: La participación en plusvalía en la ciudad de Cali. Revista Científica Guillermo de Ockham, 8(1), 39-48. https://revistas.usb.edu.co/index.php/GuillermoOckham/article/ download/552/353/

Hui, E. C-M., Ho, V. S-M. \& Ho, D. K-H. (2004). Land value capture mechanisms in Hong Kong and Singapore: A comparative analysis. Journal of Property Investment and Finance, 22(1), 76-100. https://doi.org/10.1108/14635780410525153

Instituto Nacional de Estadística y Geografía (INEGI). (2014). Censos Económicos 2014. https:// www.inegi.org.mx/programas/ce/2014/

Instituto Nacional de Estadística y Geografía (INEGI). (2019). Finanzas Públicas Estatales y Municipales. https:/www.inegi.org.mx/programas/finanzas/

Jaramillo, S. (2011). Fundamentos económicos de la participación en plusvalías. En S. Jaramillo, V. Moncayo \& O. Moncayo (Eds.), Plusvalias urbanas. Fundamentos económicos y jurídicos (pp. 59-210). Universidad Externado de Colombia, Bogotá.

Ley de Desarrollo Territorial, 388 de 1997. Colombia. http://recursos.ccb.org.co/ccb/pot/PC/ files/ley388.html

Maldonado, M. (2007). Recuperación de plusvalías. En M. Smolka \& L. Mullahy (Eds.), Perspectivas urbanas: Temas críticos en politicas de suelo en América Latina (pp. 195258). Lincoln Institute of Land Policy.

Mathur, S. (2019). An evaluative framework for examining the use of land value capture to fund public transportation projects. Land Use Policy, 86, 357-364. https://www. sciencedirect.com/science/article/abs/pii/S0264837718318945

McAllister, S., Shepherd, E. \& Wyatt, P. (2018). Policy shifts, developer contributions and land value capture in London 2005-2017. Land Use Policy, 78, 316-326. https://www. sciencedirect.com/science/article/abs/pii/S0264837717315624

Medina, V. D. (2013). Rentas urbanas y alternativas de recuperación estatal. Aproximaciones al caso de San Carlos de Bariloche y la participación municipal en la renta diferencial. Quid, 16(3), 136-156. https://publicaciones.sociales.uba.ar/index.php/quid16/ article/view/1134 
Moncayo, V. (2011). La plusvalía urbana en Colombia: Un instrumento fiscal de afectación de la renta diferencial inmobiliaria. En S. Jaramillo, V. Moncayo \& O. Moncayo (Eds.), Plusvalías urbanas. Fundamentos económicos y jurídicos (pp. 211-329). Universidad Externado de Colombia, Bogotá.

Morell, M. (2014). Nous reptes econòmics i financers per a la planificació urbanística De la plusvàlua generalitzada a l'urbanisme de gra menut que possibiliti la creació de valor. Papers. Discursos Emergents Per A Un Non Urbanisme, 57, 97-104, https://iermb.uab. cat/wp-content/uploads/2015/10/papers-57-online1.pdf

Munévar, C. \& Hernández, N. (2017). La naturaleza jurídica de la plusvalía urbana. Un análisis desde los derechos colectivos y la función social de la propiedad. Opción. Revista de Ciencias Humanas y Sociales, 33(82), 280-297. https://dialnet.unirioja.es/ servlet/articulo? codigo $=6233628$

Munévar, C., Hernández, N. \& Cardona, A. (2018). La eficacia de la participación de la plusvalía urbana en el municipio de Manizales-Colombia. Revista Republicana, 24, 41-60. https://doi.org/10.21017/Rev.Repub.2018.v24.a39

Nohlen, D. (2000). Método Comparativo. Notas. https://www.rzuser.uni-heidelberg.de/-k95/ es/doc/diccionario_metodo-comparativo.pdf

Ochoa, M. \& Rojas, B. (2007). Participación en plusvalías: instrumento para la gestión del riesgo en el municipio de Pereira. Revista Scientia et Technica, 13(34), 625-630. https:// www.redalyc.org/articulo.oa?id=84934106

Peinado, G., Barenboim, C., Nicastro, M. \& Lagarrigue, P. (2018). Recuperación de plusvalías urbanas y sus impactos distributivos. Las compensaciones por mayor aprovechamiento urbanístico a raíz de convenios urbanísticos en la ciudad de Rosario, Argentina. Revista Sociedad y Economía, 35, 50-77. http://dx.doi.org/10.25100/sye.v0i35.7295

Piovani, J. I. \& Krawczyk, N. (2017). Los estudios comparativos: algunas notas históricas, epistemológicas y metodológicas. Educaçao \& Realidade, 42(3), 821-840. http://www. memoria.fahce.unlp.edu.ar/art_revistas/pr.8927/pr.8927.pdf

Quitián-Díaz, M. L. \& Ruiz, A. (2016). Fortalecimiento financiero municipal a través de los hechos generadores de plusvalía. Contexto, 5, 153-159. https://doi.org/10.18634/ ctxj. 5v.0i.378

Ricardo, D. (1958). Ensayo sobre la influencia del bajo precio del grano sobre los beneficios del capital. En P. Sraffa (Ed.), Obras y correspondencia de David Ricardo (Iv, pp. 3-27). Fondo de Cultura Económica.

Smolka, M. (2013). Implementación de la recuperación de plusvalías en América Latina. Políticas e instrumentos para el desarrollo urbano. Lincoln Institute of Land Policy.

Smolka, M. \& Amborski, D. (2003). Recuperación de plusvalías para el desarrollo urbano: una comparación interamericana. EURE Revista Latinoamericana de Estudios Urbano Regionales, 29(88), 55-77. http://www.eure.cl/index.php/eure/article/view/1293

Smolka, M. \& Mullahy, L. (2010). Perspectivas urbanas. En M. Smolka \& L. Mullahy, Perspectivas urbanas. Temas criticos en políticas de suelo en América Latina Perspectivas urbanas. Lincoln Institute of Land Policy.

Thorngren, C. (2000). Introducción a la contabilidad financiera (7a ed.). Pearson Educación.

Torres, M. (2006). Recuperación de la renta urbana: Una tarea ética pendiente. Revista INVI, 21(58), 42-70. http://revistainvi.uchile.cl/index.php/INVI/article/view/298/913 
Tudino, L., Mansilla, F., Cabrera, C., Nicastro, M. \& Lagarrigue, P. (2010). La gestión urbana en la Comuna de Alvear. Medio Ambiente y Urbanización, 81(1), 33-50. https://www. ingentaconnect.com/content/iieal/meda/2014/00000081/00000001/art00003

Vejarano, M. (2008). Bogotá, D.c. Primera experiencia de recuperación de la plusvalía urbana para la colectividad, en el marco de la Ley de Desarrollo Territorial. Revista ACE, 3(7), 79-106. https://upcommons.upc.edu/handle/2099/5636

Verduzco, B. \& Valenzuela, M. (2018). Captura de plusvalías, regularización de edificios y aglomeraciones turísticas en Guadalajara. Revista de Economía, Sociedad y Territorio, 28(57), 397-427. https://est.cmq.edu.mx/index.php/est/article/view/1182 